\section{A Farmacoterapia da Obesidade nos Consensos}

RESUMO

Diversos documentos têm sido publicados nos últimos anos com recomendações sobre abordagem do paciente obeso e estratégias de prevenção do excesso de peso. Uma das principais preocupações tem sido a padronização de critérios para a utilização dos agentes anti-obesidade atualmente disponiveis. A partir de uma revisão dos principais documentos de consenso publicados recentemente, discutimos a abordagem que se faz do tema farmacoterapia no Consenso Latino-Americano em Obesidade, no documento da Força Tarefa Internacional de Obesidade e na Guia Clínica para Identificação. Avaliação e Tratamento do Excesso de Peso e da Obesidade em Adultos, editado pelo National Heart, Lung and Blood Institute e pelo National institute of Health (NIH), dos Estados Unidos. (Arq Bras Endocrinol Metab 2000; 44/1: 91-94)

Unitermos: Obesidade; Consensos; Farmacoterapia; Guias; Tratamento.

\begin{abstract}
Several documents have been published in the last few years with recommendations for a rational approach of the obese patient and strategies for the prevention of weight gain. One of the main concerns has been the standardization of criteria for the use of anti-obesity agents currently available. Based on the review of the main consensus documents recently published we discuss the approach to the topic of pharmacotherapy, as seen in the Latin American Consensus on Obesity, the document published by the International Obesity Task Force and the Clinical Guidelines for Identification. Evaluation and Treatment of Overweight and Obesity in Adults, edited by the National Heart, Lung and Blood Institute and the National Institute of Health. (Arq Bras Endocrinol Metab 2000; 44/1: 91-94)
\end{abstract}

Keywords: Obesity; Consensus; Pharmacotherapy; Guidelines; Treatment.

\footnotetext{
A ESTRATÉgia TERAPÊUTICA DA OBESidade tem sofrido profundas modificações nos últimos anos. A chamada terapia ancilar evoluiu em diversos aspectos. $\mathrm{O}$ tratamento nutricional atualmente recomendado baseia-se mais em um planejamento de reeducação alimentar do que nas dietas tradicionais, que freqüentemente fracassavam em manter a perda de peso. Enfatiza-se cada vez mais a importância da atividade física, com uma intensidade que deve variar de acordo com a aptidão física de cada indivíduo. As técnicas de terapia cognitivo-comportamental foram consideravelmente aperfeiçoadas, contribuindo hoje de forma mais efetiva para a mudança dos hábitos de vida do paciente obeso $(1,10,11)$.

O uso racional dos agentes anti-obesidade é considerado atualmente um coadjuvante indispensável para um grande número de obesos, quando
}

\section{artigo original}

\author{
Walmir F. Coutinho \\ Monica D. Cabral
}

\author{
Departamento de Endocrinologia da \\ Pontifücia Universidade \\ Católica do Rio de Janeiro; $e$ \\ Instituto Estadual de Diabetese \\ Endocrinologia do Rio de Janeiro, RJ.
}


já existe um risco importante para a saúde $(5,8)$. A definição dos critérios para sua utilização tem sido uma das principais preocupações dos responsáveis pela elaboração dos documentos de consenso que têm sido utilizados como guias de referência clínica para a abordagem do paciente obeso.

Um dos primeiros documentos a estabelecer parâmetros para nortear a abordagem do paciente obeso foi o da Academia Nacional de Ciências dos Estados Unidos, publicado em 1995 sob o título "Pesando as Opções" (12). Foi um instrumento útil na promoção da abordagem passo a passo recomendada pela Associação Americana de Obesidade. A primeira recomendação européia foi editada pela Scottish Intercollegiate Guidelines Network em 1996, relatando o uso da farmacoterapia de longo prazo no tratamento da obesidade e desenvolvendo uma abordagem baseada em evidências para as recomendações terapêuticas (9). Em 1997, a Organização Mundial de Saúde publicou os resultados de um trabalho internacional, com o título iObesity: preventing and managing the global epidemic". (7). O "National Heart, Lung and Blood Institute" e o National Institute of Health (NIH), dos Estados Unidos, publicaram em $1998 \mathrm{seu}$ documento entitulado "Clinical Guidelines for Treatment of Adult Obesity" (3). No mesmo ano foi editado c publicado o Conscnso Latino-Americano de Obesidade, no qual trabalharam representantes de 12 países latino-americanos (4).

Analisaremos 3 destes documentos: o da Força Tarefa Internacional de Obesidade (International Obesity Task Force - IOTF), o Consenso Latino-Americano de Obesidade (CLAO) e a Guia Clínico para a Identificação, Avaliação e Tratamento de Adultos com Excesso de Peso e Obesidade (Clinical Guidelines on the Identification, Evaluation and Treatment of Overweight and Obesity in Adults) editado pelo National Heart, Lung and Blood Institute / National Institute of Health (NIH), dos Estados Unidos.

\section{O QUE DIZEM OS CONSENSOS}

\section{Princípios da farmacoterapia}

Os consensos são unânimes em recomendar que a farmacoterapia seja sempre utilizada em conjunto com um programa de mudança de estilo de vida, como um auxiliar na mudança de hábitos alimentares e prática regular de atividades físicas.

É ressaltado que os medicamentos só devem ser utilizados sob supervisão médica e após uma avaliação cuidadosa da relação risco-beneficio para cada paciente especificamente.

O documento latino-americano alerta para a possibilidade de recuperação do peso quando se suspende o medicamento e sugere que o uso deve ser prolongado, salientando, entretanto, que os fármacos atualmente disponíveis ainda não foram avaliados por longos períodos.

O documento do NIH avalia, em 6 meses, o prazo necessário para atingir-se a perda de peso recomendável, que deve ser em torno de $10 \%$, com uma velocidade aproximada de meio a um quilograma por semana. A manutenção do peso é apontada como prioridade após os 6 primeiros meses de tratamento. $O$ documento adverte, ainda, que a idade avançada não deve ser, por si só, uma contra-indicação para o tratamento.

O documento latino-americano lembra também que o objetivo do tratamento da obesidade não deve restringir-se à redução do peso, mas, principalmente, direcionar-se à diminuição do risco de morbiletalidade. A perda de peso recomendada é de 5 a $10 \%$, para que se evitem os fracassos terapêuticos decorrentes de expectativas pouco realistas.

\section{Medicamentos recomendados}

O documento da IOTF classifica os agentes disponíveis em 3 grupos: o primeiro reúne os medicamentos com ação exclusivamente central; o segundo é o de ação periférica e o terceiro é o de ação periférica e central (Tabela 1 ).

Destaca-se, em seguida, a informação sobre a retirada da fenfluramina e da dexfenfluramina do mercado em setembro de 1997.

A fluoxetina e a sertralina são citadas como úteis no tratamento de estados depressivos associados com a obesidade, mas adverte-se que não podem ser consideradas agentes anti-obesidade.

$O$ consenso latino-americano cita também as doses recomendadas para cada medicamento (Tabela 2).

Tabela 1. Medicamentos e seus modos de ação principal.

Modo de ação principal

\section{Ação central}

Noradrenérgica

Serotoninérgica

Serotoninérgica e

noradrenérgica

Serotoninérgica e

noradrenérgica

Açōo periférica

Inibidor da lipase

Ação periférica e central

Termogênico e anorético

\section{Nome do medicamento}

Fentermina

Dexfenfluramina/fenfluramina

Fenfluramina + fentermina

Sibutramina

Orlistat

Efedrina/ cafeína 
Tabela 2. Doses recomendadas para cada medicamento, segundo o consenso latino-americano.

\begin{tabular}{lccc}
\hline Grupo & Medicamento & $\begin{array}{c}\text { Dose } \\
\text { Minima (mg) }\end{array}$ & $\begin{array}{c}\text { Dose } \\
\text { Máxima }\end{array}$ \\
Catecolaminérgicos & Fentermina & 30 & 60 \\
& Femproporex & 20 & 50 \\
& $\begin{array}{c}\text { Anfepramona } \\
\text { Mazindol }\end{array}$ & 40 & 120 \\
Serotoninérgicos & $\begin{array}{c}\text { Fenilpropanolamina } \\
\text { Fluoxetina }\end{array}$ & 50 & 3 \\
Termogênicos & Sertralina & 50 & 75 \\
& Efedrina & 50 & 150 \\
Inibidor de absorção & Cafeína & 100 & 75 \\
de gorduras & Aminofilina & 300 & 450 \\
Serotinérgico & Orlistat & $120 \times 3$ & $120 \times 3$ \\
Catecolaminérgico & Sibutramina & 10 & 20 \\
\hline
\end{tabular}

\section{Tratamentos não recomendados}

O documento da IOTF cita como "medicamentos não apropriados para o tratamento da obesidade" os diuréticos, a gonadotrofina coriônica, a anfetamina, a dexanfetamina e a tiroxina. Adverte-se que a metaformina e a acarbose podem ser úteis no tratamento de pacientes diabéticos obesos, mas não têm eficácia comprovada para o obeso não diabético. A fluoxetina, a sertralina e outros inibidores seletivos de recaptação da serotonina são apenas indicados para o tratamento de obesos com síndromes depressivas.

O documento de Consenso Latino-Americano cita ainda outras formas de tratamento muito utilizadas no nosso meio como não recomendadas para o tratamento da obesidade, pela absoluta falta de evidências científicas de eficácia. Estão citadas neste grupo a acupuntura, os cremes para celulite e obesidade, a fitoterapia, a mesoterapia, a yoga, a hipnoterapia, as fórmulas magistrais ditas naturais, os diuréticos e os laxativos. Os hormônios propostos como agentes terapêuticos da obesidade são analisados em um capítulo a parte, no qual se conclui que, apesar de alguns destes hormônios poderem ser benéficos para os pacientes obesos, não podem ser considerados eficazes como agentes anti-obesidade.

\section{O QUE NĀO DIZEM OS CONSENSOS}

Diversos aspectos relevantes para a abordagem clínica do paciente obeso são omitidos nestes documentos, ou aparecem apenas mencionados sem recomendações específicas sobre a conduta recomendada.

Pouco se fala, por exemplo, sobre a relevância da medida da circunferência da cintura na avaliação do risco do paciente obeso. O documento da IOTF cita um estudo holandês, que determinou um ponto de corte de $80 \mathrm{~cm}$ de cintura para mulher e $94 \mathrm{~cm}$ para homens como indicadores de risco metabólico.

$\mathrm{O}$ único dos 3 documentos a mencionar doses mínimas e máximas para os agentes anti-obesidade é o Latino-Americano. São citados como medicamentos úteis a fluoxetina e a sertralina, anti-depressivos com provável efeito de redução de ingesta alimentar. O documento da IOTF adverte, entretanto, que só deveriam ser utilizados em pacientes com síndromes depressivas. Não se discute, tampouco, a importância de se fazer uma pré-seleção adequada de pacientes para classes específicas de agentes antiobesidade. Os pacientes com transtorno da compulsão alimentar periódica, por exemplo, provavelmente vão necessitar de um medicamento com perfil farmacológico adequado para o controle dos episódios compulsivos (2).

Outro aspecto que talvez merecesse considerações é o critério de precedência na indicação dos medicamentos. Pelo risco de que os anorexígenos causem dependência, talvez fosse justificável reservar seu uso para os pacientes que não respondam aos agentes anti-obesidade mais seguros.

\section{O futuro dos consensos}

Espera-se para os próximos anos um grande número de novas publicações com guias clínicas orientando a abordagem do paciente obeso. A necessidade de atualização dos consensos é constante, já que as evidências científicas sobre as quais eles se fundamentam têm atravessado mudanças cada vez mais rápidas, com novas alternativas terapêuticas surgindo e sendo reavaliadas freqüentemente.

O Consenso Latino-Americano de Obesidade é um documento aberto, sujeito a revisões periódicas a partir de sugestões dos membros de seus comitês permanentes ou de contribuições de qualquer profissional de saúde ou entidade científica interessada no estudo da obesidade. Está prevista para junho do próximo ano uma tiragem com 100.000 cópias da versão resumida do documento, para ser distribuída para profissionais de saúde em todo o país.

\section{REFERÊNCIAS}

1. Brownell KD. The LEARN Program for weight control ( $7^{\text {th }}$ ed). Dallas TX: American Health Publishing Company. 1997.

2. Bruce B, Wilfley D. Binge eating among the overweight population: A serious and prevalent problem. J Am Dief Assoc 1996:96:58-61. 
3. Clinical Guidelines on the identification, evaluation, and treatment of overweight and obesity in aduits. National Heart, Lung, and Blood Institute (NHLBI), Clinical Guidelines for Obesity, 1998.

4. Consenso Latino-Americano em Obesidade. Rio de Janeiro, Brasil, 1998

5. Goldstein D. Potvin J. Long-term weight loss: the effect of pharmacological agents. Am J Clin Nut 1994;647-57.

6. National Task Force on the prevention and treatment of obesity. Long term pharmacotherapy in the management of obesity. JAMA 1996;276:1907-5.

7. Report of a WHO Consultation on Obesity: Obesity - preventing and managing the global epidemic. Geneva: World Health Organization, 1997.

8. Spitz A, Heymsfeld S, Brank RC. Drug therapy for obesity: clinical considerations. Endoc Prac 1995:1:274-9.

9. The management of obese patients in Scotland: Integrating a new approach in primary health care with a national prevention and management strategy. Eddinburg: Scottish Intercollegiate Guidelines Network, 1996.

10. Wadden TA. New goals of obesity treatment: a healthier weight and other ideals. Prim Psyc J 1998:5:45-54.

11. Wing RR. Behavioral approach to the treatment of obesity. In: Bray $G A$, Bouchard $C \Theta$ James WPT. Handbook of Obesity, New York: Marcel Dekker, inc 1998:855-73.

12. Weighing the options. Wasghinton, DC: National Academy Press, 1995.

\section{Endereço para correspondência:}

Walmir Coutinho

Av. Arnando Lombardi no 800 - Sl. 238

Barra da Tijuca

22640-000 - Rio de Janeiro 